\title{
On the Quantum Reconstruction of the Riemann zeros
}

\author{
Germán Sierra \\ Instituto de Física Teórica, CSIC-UAM, Madrid, Spain
}

(Dated: October, 2007)

\begin{abstract}
We discuss a possible spectral realization of the Riemann zeros based on the Hamiltonian $H=x p$ perturbed by a term that depends on two potentials, which are related to the Berry-Keating semiclassical constraints. We find perturbatively the potentials whose Jost function is given by the zeta function $\zeta(\sigma-i t)$ for $\sigma>1$. For $\sigma=1 / 2$ we find the potentials that yield the smooth approximation to the zeros. We show that the existence of potentials realizing the zeta function at $\sigma=1 / 2$, as a Jost function, would imply that the Riemann zeros are point like spectrum embedded in the continuum, resolving in that way the emission/spectral interpretation.

PACS numbers: 02.10.De, 05.45.Mt, 11.10.Hi
\end{abstract}




\section{INTRODUCTION}

One of the most important problems in Mathematics is the proof of the Riemann hypothesis $(\mathrm{RH})$ which states that the non trivial zeros of the classical zeta function all have real part equal to $1 / 2$ [1, 2]. The importance of this conjecture lies in its connection with the prime numbers. If the $\mathrm{RH}$ is true then the statistical distribution of the primes will be constrained in the most favorable way. According to Michael Berry the truth of the RH would mean that "there is music in the primes" [3, 4]. Otherwise, in the words of Bombieri, the failure of the $\mathrm{RH}$ would create havoc in the distribution of the prime numbers [5].

In so far, the proof of the $\mathrm{RH}$ has resisted the attempts of many and most prominent mathematicians and physicist for more than a century, which explains in part its popularity [6, 7, 8]. For these and other reasons the RH stands as one of the most fundamental problems in Mathematics for the XXI century with possible implications in Physics. In fact, physical ideas and techniques could probably be essential for a proof of the RH [9, 10]. This suggestion goes back to Polya and Hilbert which, according to the standard lore, proposed that the imaginary part of the non trivial Riemann zeros are the eigenvalues of a self-adjoint operator $H$ and hence real numbers. In the language of Quantum Mechanics the operator $H$ would be nothing but a Hamiltonian whose spectrum contains the Riemann zeros.

The Polya-Hilbert conjecture was for a long time regarded as a wild speculation until the works of Selberg in the 50's and those of Montgomery in the 70's. Selberg found a remarkable duality between the length of geodesics on a Riemann surface and the eigenvalues of the Laplacian operator defined on it [11]. This duality is encapsulated in the so called Selberg trace formula, which has a strong similarity with the Riemann explicit formula relating the zeros and the prime numbers. The Riemann zeros would correspond to the eigenvalues, and the primes to the geodesics. This classical versus quantum version of the primes and the zeros was also at the heart of the so called Quantum Chaos approach to the RH (see later). Quite independently of the Selberg work, Montgomery showed that the Riemann zeros are distributed randomly and obeying locally the statistical law of the Random Matrix Models (RMM) [12]. The RMM were originally proposed to explain the chaotic behaviour of the spectra of nuclei but it has applications in another branches of Physics, specially in Condensed Matter [13]. There are several universality classes of random matrices, and it turns out that the one related to the Riemann zeros is the gaussian unitary ensemble 
(GUE) associated to random hermitean matrices. Montgomery analytical results found an impressive numerical confirmation in the works of Odlyzko in the 80's, so that the GUE law, as applied to the Riemann zeros is nowadays called the Montgomery-Odlyzko law [14].

It is worth to mention that the prime numbers, unlike the Riemann zeros, are distributed almost at random over the set of integers. Indeed, it is believed that one can find arbitrary pairs of nearby odd numbers $n, n+2$, as well as pairs arbitrarily separated. The only thing known about the distribution of the primes is the Gauss law according to which the $\mathrm{n}^{\text {th }}$ prime $p_{n}$ behaves asymptotically as $p_{n} \sim n / \log n$ [1]. This statement is called the Prime Number Theorem proved by Hadamard and de la Valle-Poussin in 1896. If the RH is true then the deviation from the Gauss law is of order $\sqrt{n} \log n$. The analogue of the Gauss law for the imaginary part of the Riemann zeros (called it $E$ ) is given by the Riemann law where the $\mathrm{n}^{\text {th }}$-zero behaves as $E_{n} \sim 2 \pi n / \log n$. Hence, large prime numbers are progressively scarced, while large Riemann zeros abound.

An important hint suggested by the Montgomery-Odlyzko law is that the Polya-Hilbert Hamiltonian $H$ must break the time reversal symmetry. The reason being that the GUE statistics describes random Hamiltonians where this symmetry is broken. A simple example is provided by materials with impurities subject to an external magnetic field, as in the Quantum Hall effect.

A further step in the Polya-Hilbert-Montgomery-Odlyzko pathway was taken by Berry [3, 4]. who noticed a similarity between the formula yielding the fluctuations of the number of zeros, around its average position $E_{n} \sim 2 \pi n / \log n$, and a formula giving the fluctuations of the energy levels of a Hamiltonian obtained by the quantization of a classical chaotic system [15]. The comparison between these two formulas suggests that the prime numbers $p$ correspond to the isolated periodic orbits whose period is $\log p$. In the Quantum Chaos scenario the prime numbers appear as classical objects, while the Riemann zeros are quantal. This classical/quantum interpretation of the primes/zeros is certainly reminiscent of the one underlying the Selberg trace formula mentioned earlier. One success of the Quantum Chaos approach is that it explains the deviations from the GUE law of the zeros found numerically by Odlyzko. The similarity between the fluctuation formulas described above, while rather appealing, has a serious drawback observed by Connes which has to do with an overall sign difference between them [16]. It is as if the periodic orbits were missing in the underlying classical chaotic dynamics, a fact that is difficult to understand physically. This and other 
observations lead Connes to propose a rather abstract approach to the $\mathrm{RH}$ based on discrete mathematical objects known as adeles [16]. The final outcome of Connes work is a trace formula whose proof, not yet found, amounts to that of a generalized version of the RH. In Connes approach there is an operator, which plays the role of the Hamiltonian, whose spectrum is a continuum with missing spectral lines corresponding to the Riemann zeros. We are thus confronted with two possible physical realizations of the Riemann zeros, either as point like spectra or as missing spectra in a continuum. Later on we shall see that both pictures can be reconciled in a QM model having a discrete spectra embedded in a continuum.

\section{SEMICLASSICAL APPROACH}

In 1999 Berry and Keating on one hand [17, 18], and Connes on the other [16], proposed that the classical Hamiltonian $H=x p$, where $x$ and $p$ are the position and momenta of a 1D particle, is closely related to the Riemann zeros. The classical trayectories of the particle are hyperbolas in the phase space $(x, p)$, hence one should not expect a discrete spectrum even at the semiclassical level (see fig. 1. To overcome this difficulty, Berry and Keating proposed to restrict the phase space to those points where $|x|>l_{x}$ and $|p|>l_{p}$, with $l_{x} l_{p}=2 \pi \hbar$. The number of semiclassical states, $\mathcal{N}(E)$, with energy between 0 and $E$ is given by the allowed area in phase space divided by $h=2 \pi(\hbar=1)$ (see fig. 11)

$$
\mathcal{N}(E)=\frac{E}{2 \pi}\left(\log \frac{E}{2 \pi}-1\right)+1
$$

Eq.(11) coincides asympotically with the smooth part of the formula that gives the number of Riemann zeros in the same interval. This result is really striking given the simplicity of the Hamiltonian and the asumptions made. On the other hand Connes started on the same classical Hamiltonian $H=x p$ but constrained the phase space to those trayectories satisfying $|x|<\Lambda,|p|<\Lambda$, with $\Lambda$ a cutoff which is sent to infinity at the end of the calculation. The number of semiclassical states is given by

$$
\mathcal{N}(E)=\frac{E}{\pi} \log \Lambda-\frac{E}{2 \pi}\left(\log \frac{E}{2 \pi}-1\right)
$$

The first term describes a continuum of states in the limit $\Lambda \rightarrow \infty$, while the second term coincides with minus the average position of the Riemann zeros (1). This result lead Connes 


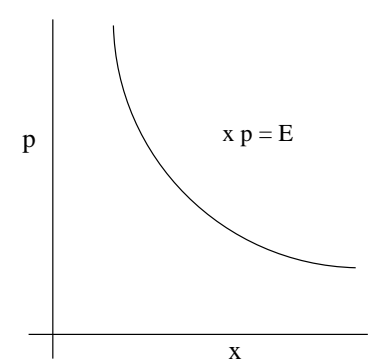

(1a)

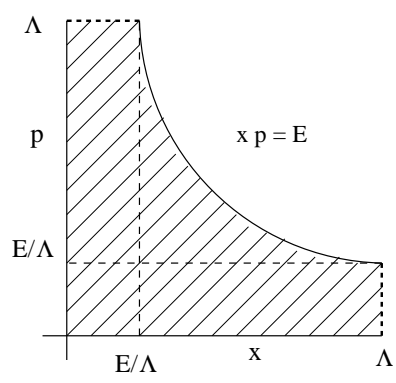

(1c)

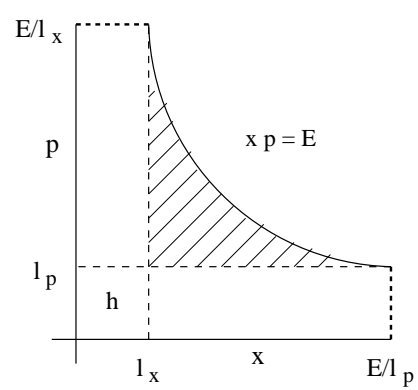

(1b)

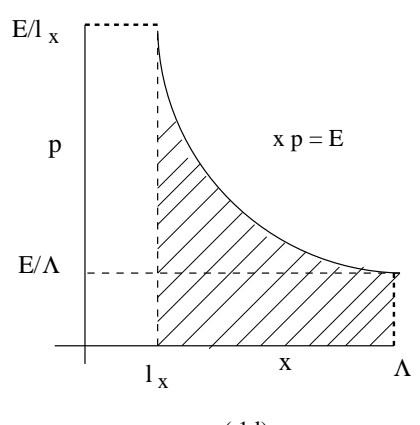

(1d)

FIG. 1: 1a) a classical trayectory of the Hamiltonian $H=x p$. The regions in shadow are the allowed phase space of the semiclassical regularizations of $H=x p$ considered by: 1b) Berry and Keating, 1c) Connes and 1d) Sierra.

to propose the missing spectral interpretation of the Riemann zeros described earlier.

A third possible regularization of the xp model, proposed in references [19, 20], is that $l_{x}<x<\Lambda$, which leads to the following counting of semiclassical states,

$$
\mathcal{N}(E)=\frac{E}{2 \pi} \log \frac{\Lambda}{l_{x}}
$$

This result agrees with the asymptotic part of (2), meaning that there is a continuum spectrum but the possible connection with the Riemann zeros is lost. The main advantage of the latter regularization is that it arises from a consistent quantization of $H=x p$ unlike the two previous semiclassical regularizations.

\section{QUANTIZATION OF $x p$ AND $1 /(x p)$}

The classical Hamiltonian $H=x p$ can be consistently quantized in two cases depending on the choice of the domain in the $x$ coordinate: 1) $0<x<\infty$ and 2) $l_{x}<x<\Lambda$ [20, 21]. 
In the first case $H$ is essentially self-adjoint, while in the second it admits a one parameter self-adjoint extension. We shall consider the latter case. To do so one first define the normal ordered operator [17]

$$
H_{0}=\frac{1}{2}(x p+p x)=-i\left(\frac{d}{d x}+\frac{1}{2}\right)
$$

where $p=-i d / d x$. The formal eigenfunctions of (44) are

$$
\psi_{E}(x)=\frac{C}{x^{1 / 2-i E}}, \quad 1<x<N
$$

where we have normalized $l_{x}=1$ and $\Lambda=N$. One can show that (4) is self-adjoint if the wave functions satisfy the boundary condition

$$
e^{i \theta} \psi_{E}(1)=N^{1 / 2} \psi_{E}(N)
$$

where the angle $\theta$ parameterizes the self-adjoint extension of $H$. Imposing (6]) on (5) yields

$$
N^{i E}=e^{i \theta}
$$

which determines the eigenvalues of $H$

$$
E_{n}=\frac{2 \pi}{\log N}\left(n+\frac{\theta}{2 \pi}\right), \quad n=0, \pm 1, \ldots
$$

This equation agrees with the semiclassiclassical result (3). In the particular case where $\theta=\pi$, the spectrum (8) becomes symmetric around 0 . Notice that the zero eigenvalue is excluded. Another way to derive this result is by considering the inverse of the operator (4). This can be done as follows,

$$
H_{0}=\frac{1}{2}(x p+p x)=x^{\frac{1}{2}} p x^{\frac{1}{2}} \rightarrow H^{-1}=x^{-\frac{1}{2}} p^{-1} x^{-\frac{1}{2}}
$$

where $x^{1 / 2}$ is well defined since $x>1$. The inverse of the momenta operator $p$ is given by the $1 \mathrm{D}$ Green function

$$
p^{-1}=\frac{i}{2} \frac{\operatorname{sign}\left(x-x^{\prime}\right)}{\sqrt{x x^{\prime}}},
$$

where $\operatorname{sign}\left(x-x^{\prime}\right)$ is the sign function. The Schroedinger equation associated to (9) is 


$$
\frac{i}{2} \int_{1}^{N} d x^{\prime} \frac{\operatorname{sign}\left(x-x^{\prime}\right)}{\sqrt{x x^{\prime}}} \psi_{E}\left(x^{\prime}\right)=\frac{1}{E} \psi_{E}(x) .
$$

which in terms of the new wave function

$$
\phi_{E}(x)=x^{-\frac{1}{2}} \psi_{E}(x)
$$

becomes

$$
x \phi_{E}(x)-\frac{i E}{2} \int_{1}^{N} d x^{\prime} \operatorname{sign}\left(x-x^{\prime}\right) \phi_{E}\left(x^{\prime}\right)=0
$$

whose solution is

$$
N^{i E}=-1, \quad \phi(x)=\frac{C}{x^{1-i E}}
$$

Hence we recover eqs.(5) and (7) in the particular case where $\theta=\pi$. On the other hand, eq.(13) looks as the eigenvalue equation of yet another Hamiltonian that we shall discuss next.

\section{RELATION WITH THE RUSSIAN DOLL BCS MODEL}

In reference [22, 23, 24] it was defined an extension of the BCS model of superconductivity, called the Russian Doll model, whose Hamiltonian, when restricted to the one body case becomes

$$
H_{R D}\left(x, x^{\prime}\right)=\varepsilon(x) \delta\left(x-x^{\prime}\right)-\frac{1}{2}\left(g+i h \operatorname{sign}\left(x-x^{\prime}\right)\right)
$$

where $\varepsilon(x)$ represents the energies of pairs of electrons occupying time reversed states in the band $1<x<N, g$ is the standard BCS coupling constant and $h$ a coupling that breaks the time reversal symmetry. The eigentates and eigenfunctions of (15) are given by

$$
\left(\frac{N-E_{R D}}{1-E_{R D}}\right)^{i h}=\frac{g+i h}{g-i h}, \quad \phi(x)=\frac{C}{\left(x-E_{R D}\right)^{1-i h}}
$$

Comparing (16) with (14), one obtains the following map between the eigenstates of the xp model and the RD model 


$$
\begin{gathered}
E_{R D}=0 \leftrightarrow E \neq 0 \\
h \leftrightarrow E \\
\frac{h}{g} \leftrightarrow \tan (\theta / 2) \\
\phi \leftrightarrow x^{-1 / 2} \psi_{E}
\end{gathered}
$$

in the particular case where $g=0$ and then $\theta=\pi$. One can add a $g$ coupling term in the definition (9) of the operator $H^{-1}$, in which case the correspondence between the RD model and the xp model will cover all the self-adjoint extensions of $x p$. The RD model provides an example where the renormalization group, instead of ending at fixed points, run in cycles $[25,26,27]$. In the RD model, the coupling that runs periodically under the RG is $g$, with a period equal to $2 \pi / h$, while the coupling $h$ remains invariant. This fact in turn implies the existence of several bound states whose number is given by $n=2 \pi / h \log N$. If we replace $h$ by $E$, the latter equation becomes $n=2 \pi / E \log N$ which coincides with the number of eigentates of the xp model.

Incidentally, we would like to mention that the field theory realizations of the cyclic Renormalization Group, of references [28, 29, 30], are at the origin of LeClair's approach to the RH [31]. In this reference the zeta function on the critical strip is related to the quantum statistical mechanics of non-relativistic, interacting fermionic gases in $1 \mathrm{~d}$ with a quasi-periodic two-body potential. This quasi-periodicity is reminiscent of the zero temperature cyclic RG of the quantum mechanical Hamiltonian of [20], but the general framework of both works is different.

\section{V. $\quad H_{0}=x p$ WITH INTERACTIONS: GENERAL MODEL}

The previous results establish an interesting correspondence between two apparently different models which also suggests a way to add interactions to the xp model. Indeed, the interacting term of the RD Hamiltonian (15) that is proportional to the coupling constant $g$ is basically a proyector operator $|B C S\rangle\langle B C S|$, with wave function $\langle x \mid B C S\rangle=1 \forall x$. As we said above, adding that term to the inverse Hamiltonian (9) would give rise to a $\theta \neq \pi$

term associated to the self-adjoint extensions of xp. Instead we want to add an interacting term that reflects the existence of two boundaries in the BK regularization of the xp model. 
The simplest possibility is to define

$$
H^{-1}=H_{0}^{-1}+\frac{i}{2}\left(\left|\psi_{a}\right\rangle\left\langle\psi_{b}|-| \psi_{b}\right\rangle\left\langle\psi_{a}\right|\right)
$$

where $\psi_{a, b}$ are two wave functions whose properties will be specified below. The matrix elements of (18) read

$$
H^{-1}\left(x, x^{\prime}\right)=\frac{i}{2} \frac{\operatorname{sign}\left(x-x^{\prime}\right)+a(x) b\left(x^{\prime}\right)-a\left(x^{\prime}\right) b(x)}{\sqrt{x x^{\prime}}}, 1<x, x^{\prime}<N
$$

where

$$
\psi_{a}(x)=\frac{a(x)}{x^{1 / 2}}, \quad \psi_{b}(x)=\frac{b(x)}{x^{1 / 2}}
$$

are real functions, which guarantee that $H^{-1}$ is a hermitean and antisymmetric matrix, so that its eigenvalues are pairs of real numbers $E,-E \neq 0$. A simplified version of (19) is obtained by choosing $b(x)=1$. The latter model will be denoted as type I, while the former as type II. For these models to be well defined in the limit $N \rightarrow \infty$ we impose the following normalization conditions

$$
\begin{aligned}
& \left|\int_{1}^{\infty} d x \frac{f(x)^{n}}{x}\right|<\infty,(n=1,2), \\
& f= \begin{cases}a & \text { type I } \\
a, b & \text { type II }\end{cases}
\end{aligned}
$$

A nice feature of the Hamiltonian (19) is that the Scroedinger equation is exactly solvable, the reason being that it is equivalent to a first order differential equation supplemented with a boundary condition. We shall next present the results obtained in reference [20]. First of all, the eigenenergies $E$ of (19) satisfy the equation

$$
\mathcal{F}_{N}(E)+\mathcal{F}_{N}(-E) N^{i E}=0
$$

where $\mathcal{F}_{N}(E)$ is a Jost like function whose expression will given below in the limit $N \rightarrow \infty$. In that limit the eigenfunctions of the type II model are given by

$$
\psi_{E}(x)=\frac{1}{x^{1 / 2-i E}}\left[C_{\infty}+\int_{x}^{\infty} d y y^{-i E}\left(\frac{d a}{d y} B-\frac{d b}{d y} A\right)\right]
$$


where $A, B, C_{\infty}$ are integration constants that depend on $E$. In the limit $x \rightarrow \infty$ the functions $a(x), b(x)$ vanish sufficiently fast so that the wave function (23) is dominated by the first term, i.e.

$$
\lim _{x>>1} \psi_{E}(x) \sim \frac{C_{\infty}}{x^{1 / 2-i E}}
$$

It turns out that $C_{\infty}$ is given by the Jost function (up to a constant which can be taken as 1),

$$
C_{\infty}(E)=\mathcal{F}(E)
$$

Hencefore the energies where $\mathcal{F}(E)$ is non zero correspond to delocalized states which behave asymptotically as the eigenfunctions of the unperturbed Hamiltonian $H_{0}=(x p+p x) / 2$. For these states the ratio $\mathcal{F}(E) / \mathcal{F}(-E)$ gives the scattering phase shift. On the other hand, $C_{\infty}$ vanishes whenever $\mathcal{F}(E)$ does. These energies correspond to localized states with a finite norm. In summary, the spectrum of $H$ consists of a continuum formed by those energies where $\mathcal{F}(E) \neq 0$, plus a discrete part given by the real zeros of $\mathcal{F}(E)$ (see fig. 21).

Moreover, using the hermiticity of $H$ one can show from eq.(21) that $\mathcal{F}_{\infty}(E)$ does not have zeros with $\operatorname{Im}(E)>0$.

$$
\mathcal{F}(E)=0 \Rightarrow \operatorname{Im} E \leq 0
$$

The real zeros of $\mathcal{F}(E)$ correspond, as explained above, to localized states, while the complex zeros below the real axis correspond to resonances. These results are summarized in table 1.

\begin{tabular}{|c|c|c|c|}
\hline Eigenstate & $C_{\infty}$ & $\mathcal{F}(E)$ & Eigencondition \\
\hline Delocalized & $\neq 0$ & $\neq 0$ & $N^{i E}=-\frac{\mathcal{F}(E)}{\mathcal{F}(E)^{*}}$ \\
Localized & $=0$ & $=0$ & $\mathcal{F}(E)=0$ \\
\hline
\end{tabular}

Table 1.- Classification of eigenstates of the model.

Before giving the expression of $\mathcal{F}(E)$ for the type I and type II model we shall introduce some definitions. First of all let us define the Mellin transform of $a$ (similarly for $b(x)$ ).

$$
\widehat{a}(t)=\int_{1}^{\infty} d x x^{-1+i t} a(x), \quad a(x)=\int_{-\infty}^{\infty} \frac{d t}{2 \pi} x^{i t} \widehat{a}(t)
$$


Bound states

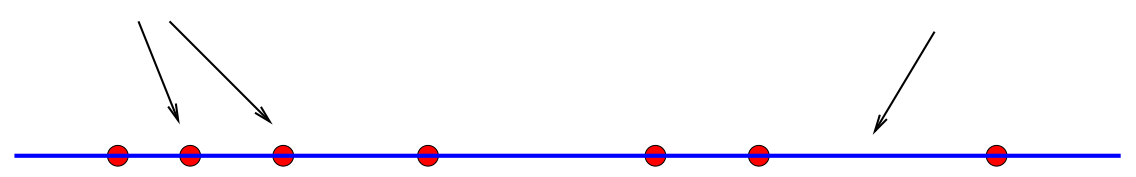

FIG. 2: Pictorial representation of the spectrum of the model. The bound states are the points where $\mathcal{F}(E)=0$, which are embedded in a continuum of scattering states.

The reality of $a(x)$ implies

$$
\widehat{a}^{*}(t)=\widehat{a}(-t), \quad t \in \mathbb{R}
$$

Condition $n=1$ in eq.(21) amounts to

$$
|\widehat{a}(0)|<\infty
$$

while condition $n=2$ in eq.(21) is equivalent to

$$
\int_{1}^{\infty} d x \frac{a(x)^{2}}{x}=\int_{-\infty}^{\infty} \frac{d t}{2 \pi}|\widehat{a}(t)|^{2}<\infty,
$$

The function $\widehat{a}(t)$ is in fact the Fourier transform of $a(x)$ in the variable $q=\log x$, which takes values in the interval $(0, \infty)$. In terms of $q, a(x)$ is a square normalizable and causal function, in which case $\widehat{a}(t)$ has interesting analytic properties by a theorem due to Titchmarsh [32]. This theorem states that under the previous conditions $\widehat{a}(t)$ is analytic in the complex upper-half plane and satisfies the formula

$$
\widehat{a}(z)=P \int_{-\infty}^{\infty} \frac{d t}{i \pi} \frac{\widehat{a}(t)}{t-z}, \quad z \in \mathbb{R}
$$

where $\mathrm{P}$ denotes the Cauchy principal value of the integral. To prove this formula one uses the fact that $\widehat{a}(t)$ has no singularities in the upper-half plane and that the contour on integration on the circle $|z|=R, \operatorname{Im} z>0$ vanishes since $\lim _{|z| \rightarrow \infty}|\widehat{a}(z)|=0$. For later purposes let us define the new function

$$
\mathbf{a}(t)=\frac{i t}{2} \widehat{a}(t)
$$

and similarly $\mathbf{b}(t)$, whose properties follow from those of $\widehat{a}(t)$ namely: 
- Reality:

$$
\mathbf{a}^{*}(t)=\mathbf{a}(-t), \quad t \in \mathbb{R}
$$

- Regularity:

$$
\lim _{t \rightarrow 0} \frac{|\mathbf{a}(t)|}{t}<\infty \Longrightarrow|\mathbf{a}(0)|=0
$$

- Normalizability

$$
\int_{-\infty}^{\infty} \frac{d t}{2 \pi} \frac{|\mathbf{a}(t)|^{2}}{t^{2}}<\infty
$$

- Analiticity

$$
\mathbf{a}(z)=P \int_{-\infty}^{\infty} \frac{d t}{i \pi} \frac{\mathbf{a}(t)}{t-z}-P \int_{-\infty}^{\infty} \frac{d t}{i \pi} \frac{\mathbf{a}(t)}{t}, \quad z \in \mathbb{R}
$$

This eq. implies that $\mathbf{a}(z)$ is an analytic function in the upper-half plane which converges towards a constant value in a circle of infinite radius given by the last term of (36).

The next definition involves the product of two analytic functions $\mathbf{f}(t)$ and $\mathbf{g}(t)$ in the upper half-plane:

$$
(\mathbf{f} \star \mathbf{g})(z)=\mathbf{f}(z) \mathbf{g}(-z)+\int_{-\infty}^{\infty} \frac{d t}{i \pi} \frac{\mathbf{f}(t) \mathbf{g}(-z)}{t-z}, \quad z \in \mathbb{R}
$$

where the integration is understood in the Cauchy sense as in eqs.(31) and (36). One can show that $(\mathbf{f} \star \mathbf{g})(z)$ is an analytic function in the upper half-plane provided $\mathbf{f}(z) \mathbf{g}(-z)$ is well behaved, which seems to be the case in all the examples we have analized. The analytic extension of $(\mathbf{f} \star \mathbf{g})(z)$ to the lower half-plane will have in general singularities. In terms of this product we shall define the function [33]:

$$
S_{\mathbf{f}, \mathbf{g}}(z)=(\mathbf{f} \star \mathbf{g})(z)-(\mathbf{f} \star \mathbf{g})(0)
$$

which satisfies the following conditions 
- Reality: if $\mathbf{f}$ and $\mathbf{g}$ verify (33) then

$$
S_{\mathbf{f}, \mathbf{g}}^{*}(z)=S_{\mathbf{f}, \mathbf{g}}(-z), \quad z \in \mathbb{R}
$$

- Regularity

$$
S_{\mathbf{f}, \mathbf{g}}(0)=1
$$

- Shuffle relation

$$
S_{\mathbf{f}, \mathbf{g}}(z)+S_{\mathbf{g}, \mathbf{f}}(-z)=2 \mathbf{f}(z) \mathbf{g}(-z)
$$

The notation "shuffle" is borrowed from the Theory of multiple zeta functions, as explained later on.

After these definitions we can finally give the expression of the Jost function $\mathcal{F}(E)$ in terms of the potentials $\mathbf{a}(t)$ and $\mathbf{b}(t)$. For the type I model it reads

$$
\mathcal{F}(t)=1+2 \mathbf{a}(t)+S_{\mathbf{a}, \mathbf{a}}(t)
$$

while for the type II model it is

$$
\mathcal{F}(t)=1-S_{\mathbf{a}, \mathbf{b}}(t)+S_{\mathbf{b}, \mathbf{a}}(t)+S_{\mathbf{a}, \mathbf{a}}(t) S_{\mathbf{b}, \mathbf{b}}(t)-S_{\mathbf{a}, \mathbf{b}}(t) S_{\mathbf{b}, \mathbf{a}}(t)
$$

From the properties of the $S$-functions one can easily derived:

- Reality:

$$
\mathcal{F}^{*}(t)=\mathcal{F}(-t) \quad z \in \mathbb{R}
$$

This condition guarantees that the ratio $\mathcal{F}(E) / \mathcal{F}(-E)$ appearing in the eigenvalue eq.(22) is indeed a phase.

- Regularity

$$
\mathcal{F}(0)=1
$$


This condition implies that the zero energy is not an eigenvalue of the Hamiltonian $H$, which was the assumptions made by defining it in terms of its inverse.

Let us next consider the two models separately.

\section{A. Type I model}

The Jost function (42) can be expressed as

$$
\mathcal{F}(t)=1+2 \mathbf{a}(t)+(\mathbf{a} \star \mathbf{a})(t)
$$

where we used that $\mathbf{a}(0)=0$, which implies

$$
(\mathbf{a} \star \mathbf{a})(0)=0 .
$$

An important consequence of eq. (46) is the positivity of the real part of $\mathcal{F}(t)$,

$$
\operatorname{Re} \mathcal{F}(t)=|1+\mathbf{a}(t)|^{2} \geq 0
$$

which imposes a strong contraint on the functions allowing a QM interpretation as Jost functions of the type I model. In particular eq.(48) excludes the zeta function $\zeta(\sigma-i t)$ for $1 / 2 \leq \sigma<1$, but not the case where $\sigma>1$ as we shall see later on.

The $\star$-product defined in eq.(37) is non commutative and non associative. Nevertheless it behaves nicely respect to the identity function 1, namely

$$
1 \star 1=1
$$

and

$$
\mathbf{a} \star 1+1 \star \mathbf{a}=2 \mathbf{a}
$$

where $\mathbf{a}$ is an analytic function in the upper half-plane satisfying eq.(36). Using these two equations, the Jost function (46) can be expressed as the square, with respect to the

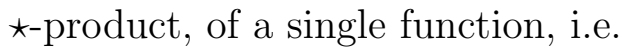

$$
\mathcal{F}=\mathbf{f} \star \mathbf{f}, \quad \mathbf{f}=1+\mathbf{a}
$$


so that $\mathbf{f}$ is the $\star$-square root, of $\mathcal{F}$. Using eq.(51) one can easily prove that $\mathcal{F}(t)$ does not have zeros in the upper half-plane. Indeed, write $\mathbf{f} \star \mathbf{f}$ as

$$
(\mathbf{f} \star \mathbf{f})(z)=\int_{-\infty}^{\infty} \frac{d t}{i \pi} \frac{\mathbf{f}(t) \mathbf{f}(-t)}{t-z}, \quad \operatorname{Im} z>0
$$

then

$$
\operatorname{Re}(\mathbf{f} \star \mathbf{f})(z)=\int_{-\infty}^{\infty} \frac{d t}{\pi} \frac{y|\mathbf{f}(t)|^{2}}{(t-x)^{2}+y^{2}}>0, \quad z=x+i y
$$

A simple but illustrative example of the theory developped so far is provided by the step potential,

$$
a(x)= \begin{cases}a_{1}, & 1<x<x_{1} \\ 0, & x_{1}<x<\infty\end{cases}
$$

which yields

$$
\mathbf{a}=\frac{a_{1}}{2}\left(x_{1}^{i t}-1\right), \quad \mathbf{a} \star \mathbf{a}=\frac{a_{1}^{2}}{2}\left(1-x_{1}^{i E}\right),
$$

and the Jost function

$$
\mathcal{F}(t)=1+\frac{a_{1}\left(2-a_{1}\right)}{2}\left(x_{1}^{i E}-1\right) .
$$

Fig. 3 shows an Argand plane representation of the real and imaginary parts of (56) for several values of $a_{1}$. For $a_{1}=1$ the function $\mathcal{F}(t)$ vanishes at the values

$$
E_{n}=\frac{(2 n+1) \pi}{\log x_{1}}, \quad n=0, \pm 1, \ldots
$$

describing an infinite number of bound states. The remaining values of $E$ correspond to delocalized states. All the zeros of $\mathcal{F}(E)$ lie on the real axis for $a_{1}=1$ and below it for $a_{1} \neq 1$.

\section{B. Perturbative solution of the type I model}

The next problem we address is: given a function $\mathcal{F}(t)$, satisfying the analyticity, reality, regularity and positivity conditions described above, which is the potential, or potentials, a $(t)$, verifying eq.(46) ? In this paragraph we shall give a perturbative method to construct 


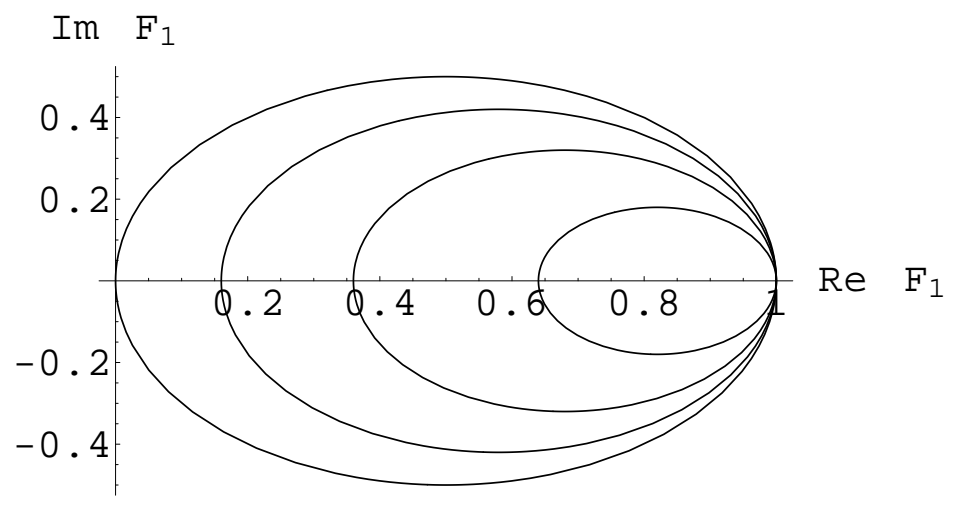

FIG. 3: Plot of the real and imaginary parts of $\mathcal{F}(E)$, as given by eq.(156), for the choices $a_{1}=$ $0.2,0.4,0.6,1$. At $a_{1}=1$ the circle passes through the origin.

one of those potential in terms of a series which converges under certain conditions placed on the function $\mathcal{F}(t)$. Let us first make the change $\mathbf{a} \rightarrow-2 \mathbf{a}$ in eq.(46) which becomes

$$
\mathbf{a}=\mathbf{g}+\mathbf{a} \star \mathbf{a}, \quad \mathrm{g}=\frac{1-\mathcal{F}}{4}
$$

Iterating (58) generates the series expansion

$$
\mathbf{a}=\mathbf{g}+\mathbf{g} \star \mathbf{g}+\mathbf{g} \star(\mathbf{g} \star \mathbf{g})+(\mathrm{g} \star \mathbf{g}) \star \mathbf{g}+\ldots
$$

At order $\mathbf{g}^{n}$ one gets all admissible bracketings, whose number is given by $C_{n-1}$, where $C_{n}$ is the Catalan number

$$
C_{n}=\frac{1}{n+1}\left(\begin{array}{c}
2 n \\
n
\end{array}\right) \stackrel{n>>1}{\rightarrow} \frac{4^{n}}{\sqrt{\pi} n^{3 / 2}}
$$

whose asymptotic behaviour is also described. To investigate the conditions for convergence of (59), let us suppose that $\mathbf{g}$ is given by the absolute convergent series

$$
\mathbf{g}(t)=\sum_{n=1}^{\infty} g_{n} n^{i t}, \quad|\mathbf{g}(t)| \leq \sum_{n=1}^{\infty}\left|g_{n}\right|<\infty
$$

Using (37) one finds 


$$
(\mathbf{g} \star \mathbf{g})(t)=\sum_{n=1}^{\infty} g_{n}^{2}+2 \sum_{n>m} g_{n} g_{m}(n / m)^{i t}
$$

so that

$$
|(\mathbf{g} \star \mathbf{g})(t)| \leq\left(\sum_{n=1}^{\infty}\left|g_{n}\right|\right)^{2}, \forall t \in \mathbb{R}
$$

Similarly one finds that

$$
|\mathbf{a}(t)| \leq \sum_{n=1}^{\infty} C_{n-1}\left(\sum_{m=1}^{\infty}\left|g_{m}\right|\right)^{n},
$$

which, according to (60), converges provided

$$
\sum_{m=1}^{\infty}\left|g_{m}\right| \leq \frac{1}{4}
$$

This condition is sufficient for convergence of the series (59) but it is not necessary. Eq.(65) implies

$$
|\mathcal{F}(t)-1| \leq 1, \quad \forall t \in \mathbb{R}
$$

However the converse is not true (i.e. eq.(66) does not imply (65)). We believe that (66) also guarantees the convergence of (59), but this guess needs to be proved. Observe also that (66) implies that $\operatorname{Re} \mathcal{F}(t) \geq 0$, which is also a necessary condition for the existence of the potential $\mathbf{a}(t)$. An application of these results is:

\section{The zeta function with $\sigma>1$}

For $\sigma>1$ let us consider the function

$$
\mathcal{F}(t)=C \zeta(\sigma-i t), \quad C=\frac{1}{\zeta(\sigma)}
$$

where the constant $C$ guarantees the normalization condition $\mathcal{F}(0)=1$. The values of the constants $g_{n}$ appearing in (61) are given by

$$
g_{1}=\frac{1-C}{4}, \quad g_{n>1}=-\frac{C}{4 n^{\sigma}}
$$


and the convergence criteria (65) yields

$$
\sum_{m=1}^{\infty}\left|g_{m}\right|=\frac{1-C}{2} \leq \frac{1}{4} \rightarrow \zeta(\sigma) \leq 2
$$

where the latter conditions is satisfied if

$$
\sigma \geq \sigma_{c}=1.72865, \quad \zeta\left(\sigma_{c}\right)=2
$$

Moreover condition (66) can be checked numerically i.e.

$$
\left|\frac{\zeta(\sigma-i t)}{\zeta(\sigma)}-1\right| \leq 1, \quad \forall \sigma>1, \quad \forall t \in \mathbb{R}
$$

so we expect that the series (59) will also converge for any value $\sigma>1$. Fig. 4 displays the real and imaginary parts of $\mathbf{a}(t)$ for the case $\sigma=2$ obtained by the sum of the first terms of eq.(59). The convergence towards a finite value is clear.

The series (59) contains also an interesting analytical structure, which can be seen from the star product of two zeta functions,

$$
\zeta(\sigma-i t)=\sum_{n=1}^{\infty} \frac{1}{n^{\sigma-i t}} \rightarrow(\zeta \star \zeta)(t)=\zeta(2 \sigma)+2 \sum_{n>m \geq 1}^{\infty} \frac{1}{n^{\sigma-i t} m^{\sigma+i t}}
$$

The double sum series of the RHS is equal to a Euler-Zagier zeta function for two variables which is a generalization of the zeta function. Multivariable versions of this function have attracted much attention in various fields, as knot theory, perturbative quantum field theory, etc (see [34], [35] and references therein).

\section{The zeta function at $\sigma=1 / 2$}

The results obtained so far suggests that the Riemann zeta function on the critical line $\zeta(1 / 2-i E)$ could perhaps be realized as the Jost function of the model. This idea is motivated by the scattering approach pionered by Faddeev and Pavlov in 1975, and has

been followed by many authors [36, 37, 38, 39]. An important result is that the phase of $\zeta(1+i t)$ is related to the scattering phase shift of a particle moving on a surface with constant negative curvature. The chaotic nature of that phase is a well known feature. Along this line of thoughts, Bhaduri, Khare and Law (BKL) maded in 1994 an analogy between resonant quantum scattering amplitudes and the Argand diagram of the zeta function $\zeta(1 / 2-i t)$, 

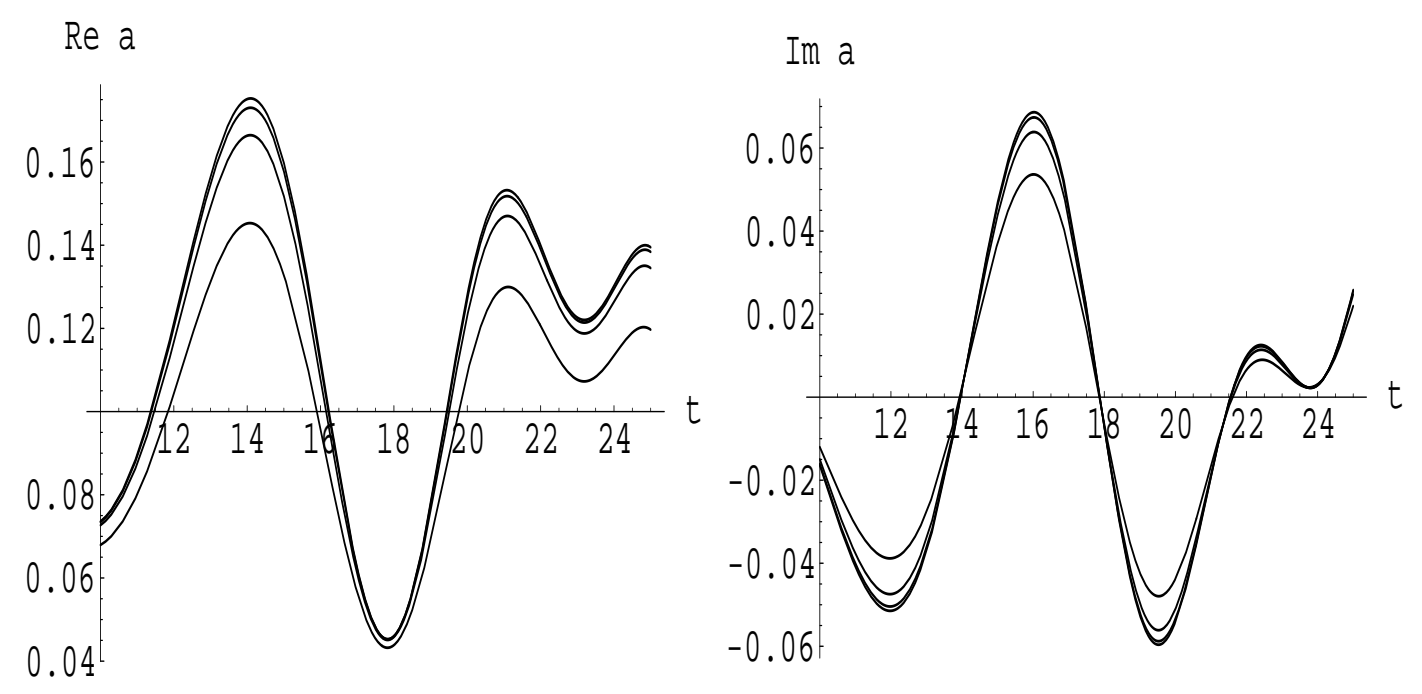

FIG. 4: Real and imaginary parts of the potential $\mathbf{a}(t)$ for $\sigma=2$ in the interval $t \in(10,25)$.

where the real part of $\zeta$ (along the $x$-axis) is plotted against the imaginary part ( $y$-axis) [40]. The diagram consists of an infinite series of closed loops passing through the origin every time $\zeta(1 / 2-i t)$ vanishes (see fig. 5). This loop structure is similar to the Argand plots of partial wave amplitudes of some physical models with the two axis being interchanged. However the analogy is flawed since the real part of $\zeta(1 / 2-i t)$ is negative in small regions of $t$, a circumstance which never occurs in those physical systems.

In fact, the loop structure of the models proposed by BKL is identical, up to a scale factor of 2, to the model with the potential (54) (see fig. (3)), where the loops representing $\mathcal{F}(E)$, for $a_{1}=1$, are circles of radius $1 / 2$, centered at $x=1 / 2$. For general models of type I, the loops are not circles but the real part of $\mathcal{F}_{1}(E)$ is always positive (see eq.(48)), and therefore they can never represent $\zeta(1 / 2-i E)$. Incidentally, this constraint does not apply to the models of type II, where $\operatorname{Re} \mathcal{F}(E)$ may become negative. This suggests that $\zeta(1 / 2-i E)$ could indeed be the Jost function $\mathcal{F}(E)$ of a type II model for a particular choice of $a$ and $b$. If this were the case then the Riemann zeros would become eigenenergies of the Hamiltonian realizing in that manner the Polya and Hilbert conjecture which may also give hints into the solution of the Riemann hypothesis. A complete answer to this problem is not yet known but we shall present below some encouraging results along this direction.

The first step is to recover quantum mechanically the smooth approximation to the 
Riemann zeros. This approximation is equivalent to the following condition

$$
1+e^{2 i \theta(E)}=0
$$

where

$$
e^{2 i \theta(E)}=\pi^{-i E} \frac{\Gamma\left(\frac{1}{4}+\frac{i E}{2}\right)}{\Gamma\left(\frac{1}{4}-\frac{i E}{2}\right)}
$$

The function $\theta(E)$ gives the phase of the zeta function, e.g.

$$
\zeta(1 / 2-i t)=Z(t) e^{i \theta(t)}
$$

while $Z(t)$ is the Riemann-Siegel zeta function which is real and even due to the duality relation satisfied by the zeta function (e.g. $\zeta(1 / 2-i t)=e^{2 i \theta(t)} \zeta(1 / 2+i t)$ ). The reason that (73) is a good approximation to the location of the zeros, can be seen in fig. 5 which plots the real and imaginary parts of $\zeta(1 / 2-i E)$. Observe that in the vicinity of a zero, the curves cut the imaginary axis where $\theta\left(E_{n}\right)=\pi(2 n-1) / 2$ so that $\cos \theta(E)=0$, which is nothing but eq.(73). The value of $E_{n}$ satisfies

$$
n=\frac{\theta\left(E_{n}\right)}{\pi}+\frac{1}{2}
$$

which asymptotically coincides with eq. (11) up to a constant term. This result can be obtained choosing the following potential in the type I model

$$
a(x)=-2 \frac{\sin (2 \pi x)}{\sqrt{x}}
$$

whose Mellin transform (27) gives

$$
\widehat{a}(t)=-\frac{2 i}{t} e^{2 i \theta(t)}+O(1 / t)
$$

up to $1 / t$ terms. The corresponding Jost function is

$$
\mathcal{F}(E)=2\left(1+e^{2 i \theta(E)}\right)+O(1 / E)
$$

whose zeros agrees with (73) asymptotically. Hence the smooth approximation to the Riemann zeros can be obtained asymptotically by the potential (77). Why this potential is able to yield this result? One may suspect that it must implement at the quantum level the BK 

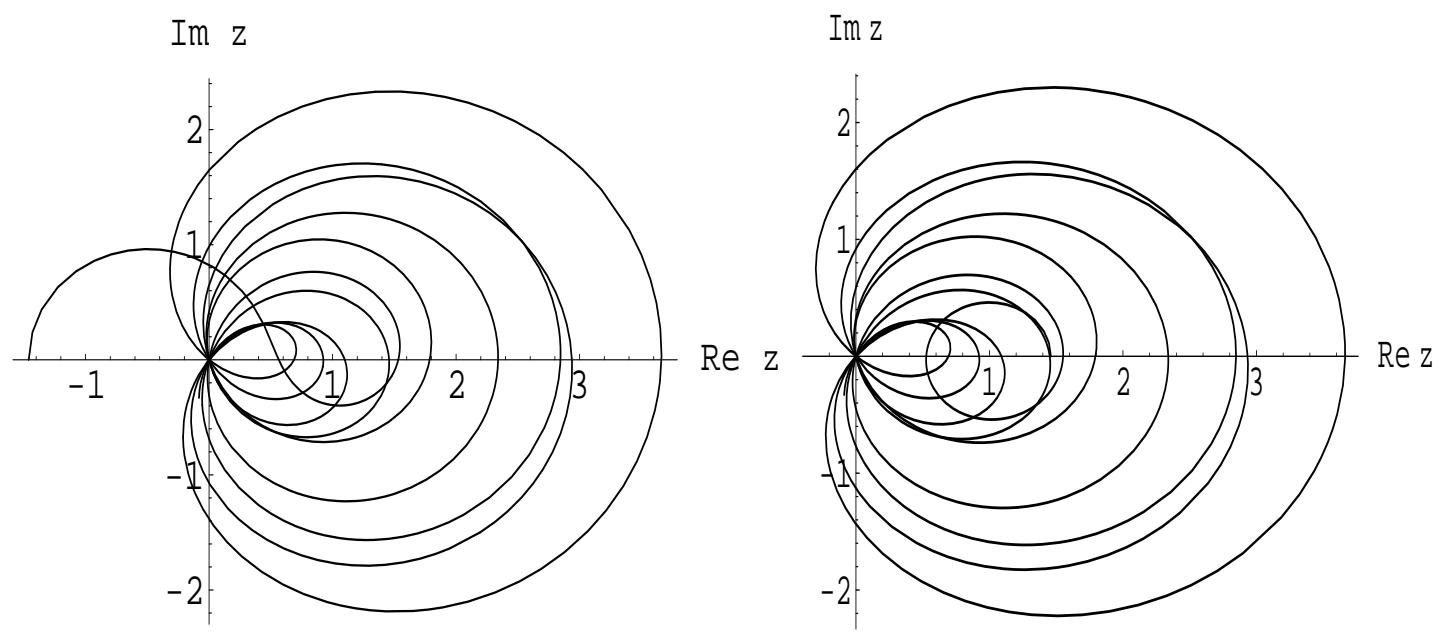

FIG. 5: Left: real and imaginary parts of $\zeta(s)$ with $s=1 / 2-i E$ and $E \in(0,50)$. Right: same as before for $\zeta_{H}(s)=(s-1) \zeta(s) / s$.

boundary conditions of the semiclassical approach. Indeed, let us show how this works in detail. The potential (77) corresponds to the wave function

$$
\psi_{a}(x)=-2 \frac{\sin (2 \pi x)}{x}
$$

which satisfies the equation

$$
H_{0}^{2} \psi_{a}(x)=\left((2 \pi x)^{2}-\frac{1}{4}\right) \psi_{a}(x),
$$

where $H_{0}=\sqrt{x} p \sqrt{x}$ is the Hamiltonian (9). Dropping $\psi_{a}$ in both sides and replacing $H_{0}$ by $x p$, one obtains a classical version of (81),

$$
(x p)^{2}=(2 \pi x)^{2}-\frac{1}{4} \Longrightarrow p= \pm 2 \pi \sqrt{1-\frac{1}{(4 \pi x)^{2}}},
$$

which describes a curve in phase space that approaches asymptotically the lines $p= \pm 2 \pi$. We shall identify these asymptotes with the BK boundary in the momenta $|p|=l_{p}$. Recall on the other hand the boundary condition $x \geq l_{x}=1$, which combined with the previous identification reproduces the Planck cell quantization condition,

$$
l_{p}=2 \pi, l_{x}=1 \Longrightarrow l_{p} l_{x}=2 \pi
$$


The BK condition $|x|>l_{x}$ has already built in into the model and this is also reflected in the state $\psi_{b}(x)=1 / \sqrt{x}$ which is concentrated near the position $l_{x}=1$. In the model we have proposed, the two BK boundary conditions are realized asymmetrically, as opposed to the semiclassical model. It would be desirable to have a more symmetric treament of them. This indeed can be done and the results will be presented elsewhere.

The next natural step is to see wether the zeta function $\zeta(1 / 2-i E)$ can be realized as the Jost function of the model. The analyticity properties of the Jost functions implies that $\mathcal{F}(E)$ must be of the form

$$
\mathcal{F}(E)=C \frac{E-i / 2}{E+i \mu} \zeta(1 / 2-i E)
$$

which does not have poles in the upper-half plane ( $C$ and $\mu>0$ are normalization constants). In fig. 5 we plot an example with $\mu=1 / 2$. Since the real part of $\zeta(1 / 2-i E)$, and thus of $\mathcal{F}(E)$, become negative in small regions of $E$ one is forced to consider the type II model with two non trivial functions $\psi_{a}$ and $\psi_{b}$. The problem of finding $\psi_{a, b}$ is rather non trivial. One can in principle fix one of them, say $\psi_{b}$, and try to solve for $\psi_{a}$ as a function of the Jost function (84) and $\psi_{b}$. In the case of the zeta function $\zeta(\sigma-i E)$, with $\sigma>1$, we were able to solve this problem perturbatively thanks to the fact that the zeta function is bounded. However for $\sigma=1 / 2$ the zeta function is unbounded which may lead to problems of convergence. In any case, it seems clear that one needs further physical insights to make progress into this difficult problem. As we said above, one needs a more symmetric treatment of the coordinates $x$ and $p$, and a clearer physical interpretation of the wave functions $\psi_{a, b}$. Another important ingredient to be implemented is the duality symmetry of the zeta function which in the present formulation of the model is not manifest but which is expected to play a central role.

In summary, we have presented in this work an interacting version of the xp Hamiltonian which may ultimately lead to a spectral realization of the Riemann zeros, as suggested long ago by Polya and Hilbert. The main ingredient of the model is the non local character of the interaction in terms of two potentials which are the quantum version of the semiclassical phase space constraints of Berry and Keating. The generic spectrum of the model consists of a continuum of eigenstates in the thermodynamic limit which may also contain bound states embedded in it. We conjecture the existence of potentials giving rise to the Riemann zeros as the discrete spectrum embedded in the continuum. If this were the case that would 
resolve the emission versus absortion spectral interpretation of the Riemann zeros. This would also open the way to a better understanding of the Riemann hypothesis. We have also pointed out the need to implement in an explicit way the duality properties of the zeta function, which implies a more symmetric treatment of the $x$ and $p$ coordinates as in the semiclassical model.

\section{ACKNOWLEDGMENTS}

I would like to thanks the organizers of the "5th International Symposium on Quantum Theory and Symmetries" held at University of Valladolid, Spain, and specially Mariano del Olmo, for the invitation to present the results of the present work. I wish also to thank Andre LeClair for the many discussions we had on our joint work on the Russian doll Renormalization Group and its relation to the Riemann hypothesis. I also thank M. Asorey, M. Berry, L.J. Boya, J. García-Esteve, J. Keating, M.A. Martín-Delgado, G. Mussardo,

J. Rodríguez-Laguna and J. Links for our conversations. This work was supported by the CICYT of Spain under the contract FIS2004-04885. I also acknowledge ESF Science Programme INSTANS 2005-2010.

[1] H.M. Edwards, "Riemann's Zeta Function", Academic Press, New York, 1974.

[2] E.C. Titchmarsh, "The Theory of the Riemann Zeta-Function", 2nd ed., Oxford University Press 1999, Oxford.

[3] M.V. Berry, in Quantum Chaos and Statistical Nuclear Physics. Eds. T.H. Seligman and H. Nishioka, Lecture Notes in Physics, No. 263, Springer Verlag, New York, 1986.

[4] M.V. Berry, "Quantum Chaology", Proc. R. Soc. Lond. A 413, 183 (1987).

[5] E. Bombieri, "Problems of the Millenium: the Riemann hypothesis", Clay Mathematics Institute (2000).

[6] P. Sarnak, "Problems of the Millenium: the Riemann hypothesis (2004)", Clay Mathematics Institute (2004).

[7] J.B. Conrey, "The Riemann Hypothesis." Not. Amer. Math. Soc. 50, 341-353, 2003.

[8] See M. Watkins at http://secamlocal.ex.ac.uk/ mwatkins 
/zeta/physics.htm for a comprehensive review on several approaches to the RH.

[9] H.C. Rosu, "Quantum hamiltonians and prime numbers", Mod. Phys. Lett. A18 (2003) 1205; quant-ph/0304139.

[10] E. Elizalde, V. Moretti, S. Zerbini, "On recent strategies proposed for proving the Riemann hypothesis", Int.J.Mod.Phys. A18 (2003) 2189-2196; math-ph/0109006.

[11] A. Selberg, "Harmonic analysis and discontinuous groups in weakly symmetric Riemannian spaces with applications to Dirichlet series", Journal of the Indian Mathematical Society 20 (1956) 47-87.

[12] H. Montgomery, "The pair correlation of zeros of the zeta function", Analytic Number Theory, AMS (1973).

[13] M.L. Mehta, "Random matrices", Elsevier Academic Press, 2004, Amsterdam.

[14] A. Odlyzko, "On the distribution of spacings between zeros of zeta functions", Math. Comp. 48, $273(1987)$.

[15] M. C. Gutzwiller "Periodic orbits and classical quantization conditions", J. Math. Phys. 12 no. 3 (1971).

[16] A. Connes, "Trace formula in noncommutative geometry and the zeros of the Riemann zeta function", Selecta Mathematica (New Series) 5 (1999) 29; math.NT/9811068.

[17] M.V. Berry and J.P. Keating, "H=xp and the Riemann zeros", in Supersymmetry and Trace Formulae: Chaos and Disorder, ed. J.P. Keating, D.E. Khmelnitskii and I. V. Lerner, Kluwer 1999.

[18] M. V. Berry and J. P. Keating, "The Riemann zeros and eigenvalue asymptotics", SIAM REVIEW 41 (2) 236, 1999.

[19] G. Sierra, "The Riemann zeros and the Cyclic Renormalization Group", J.Stat.Mech. 0512 (2005) P006; math.NT/0510572.

[20] G. Sierra,"H=xp with interaction and the Riemann zeros", Nucl. Phys. B 776, (2007) 327; math-ph/0702034.

[21] J. Twamley and G. J. Milburn, "The quantum Mellin transform”, New J. Phys. 8 (2006) 328; quant-ph/0702107.

[22] A. LeClair, J.M. Román and G. Sierra, "Russian doll Renormalization Group and Superconductivity", Phys. Rev. B69 (2004) 20505; cond-mat/0211338.

[23] A. Anfossi, A. LeClair, G. Sierra, "The elementary excitations of the exactly solvable Russian 
doll BCS model of superconductivity", J. Stat. Mech. (2005) P05011; cond-mat/0503014.

[24] C. Dunning and J. Links, "Integrability of the Russian doll BCS model", Nucl. Phys. B702 (2004) 481, cond-mat/0406234.

[25] S. D. Glazek and K. G. Wilson, "Limit cycles in quantum theories", Phys. Rev. Lett. 89 (2002) 230401, hep-th/0203088; "Universality, marginal operators, and limit cycles", Phys. Rev. B69, 094304 (2004); cond-mat/0303297.

[26] D. Bernard and A. LeClair, "Strong-weak coupling duality in anisotropic current interactions", Phys.Lett. B512 (2001) 78; hep-th/0103096.

[27] E. Braaten and H.-W. Hammer, " Universality in Few-body Systems with Large Scattering Length", Phys.Rept. 428 (2006) 259-390, cond-mat/0410417.

[28] A. LeClair, J.M. Román and G. Sierra, "Russian doll Renormalization Group and KosterlitzThouless Flows", Nucl. Phys. B675 (2003) 584; hep-th/0301042.

[29] A. LeClair, J.M. Román and G. Sierra, "Log-periodic behaviour of finite size effects in field theory models with cyclic renormalization group", Nucl. Phys. B700 [FS] (2004) 407; hep-th/0312141.

[30] A. LeClair,and G. Sierra, "Renormalization group limit-cycles and field theories for elliptic S-matrices", Theor. Exp. (2004) P08004; hep-th/0403178.

[31] A. LeClair, "Interacting Bose and Fermi gases in low dimensions and the Riemann hypothesis"; math-ph/0611043.

[32] E.C. Titchmarsh, "Introduction to the theory of Fourier integrals", Oxford University Press (1937), 2nd ed. New York.

[33] The $S_{\mathbf{f}, \mathbf{g}}(z)$ differs in a sign respect to the one considered in reference [20].

[34] S. Akiyama and Y. Tanigawa, "Multiple zeta values at non-positive integers", Ramanujan J. 5 (2001), 327-351.

[35] L. Guo and B. Zhang, "Renormalization of Multiple zeta values", math.NT/0606076.

[36] B.S. Pavlov and L.D. Faddeev, "Scattering theory and automorphic functions", Sov. Math. 3, 522 (1975), Plenum Publishing Corp. translation, N.Y;

[37] Lax and R.S. Phillips, Scattering Theory for Automorphic Functions, Princeton University Press, Princeton, 1976.

[38] M.C. Gutzwiller, "Stochastic behaviour in Quantum Scattering", Physica D7, 341 (1983).

[39] S. Joffily, "Jost function, prime numbers and Riemann zeta function", math-ph/0303014 
[40] R.K. Bhaduri, Avinash Khare, and J. Law, "Phase of the Riemann zeta function and the inverted harmonic oscillator", Physical Review E 52 no. 1 (1995) 486-491; chao-dyn/9406006. 